Radiologe 2013 · 53:1075-1076

DOI 10.1007/s00117-013-2532-3

Online publiziert: 30. November 2013

c) Springer-Verlag Berlin Heidelberg 2013

\section{W. Reith}

Klinik für Diagnostische und Interventionelle Neuroradiologie,

Universitätsklinikum des Saarlandes, Homburg/Saar

\section{Phakomatosen}

Sehr geehrte Leserinnen und Leser,

unter Phakomatosen versteht man eine Gruppe von Krankheiten und Fehlbildungen, die sich an Organen ektodermalen Ursprungs, also z. B. Nervensystem, Augen oder Haut, manifestieren. Hierzu zählen die Neurofibromatose Typ 1 und Typ 2, die tuberöse Sklerose, die retinozerebelläre Angiomatose von-Hippel-Lindau, die enzephalofaziale Angiomatosis SturgeWeber, die Ataxia teleangiectatica LouisBahr sowie das Peutz-Jeghers-Syndrom und die intestinale Polyposis mit perioraler Pigmentierung.

Der Begriff Phakomatose geht auf eine Arbeit des Arztes van der Hoeve aus dem Jahr 1920 zurück, der erstmals „linsenförmige" Veränderungen im Augenhintergrund eines Patienten mit tuberöser Sklerose beschrieben und diese Veränderungen als Phakome bezeichnet hat. 1923 beschrieb van der Hoeve die Augenveränderungen bei der Neurofibromatose Recklinghausen und der tuberösen Sklerose Bourneville als ähnliche Erscheinung und schlug den Neologismus Phakomatosen, abgeleitet von Phakos = Linse, als Sammelbezeichnung für diese Gruppe von Krankheiten vor. Bei dem Begriff handelt es sich jedoch nicht um eine wissenschaftliche Definition, sondern eher um ein Konstrukt, um Krankheitsmerkmale und -ursachen besser einzugruppieren. Aufgrund neuer Ergebnisse der molekularbiologischen Grundlagen der Erkrankungen ist der Begriff praktisch überflüssig, wird aber in der Literatur weiterhin verwendet. Phakomatosen sind pathologisch gekennzeichnet durch das Auftreten von Hamartomen in mehreren Organsystemen. Die Gruppe der Krankheiten wird aus historischen Gründen Phakomatosen genannt.

Bei der Neurofibromatose existieren 2 verschiedene Typen der Erkrankung, deren gemeinsames Hauptcharakteristikum die Bildung von Neurofibromen und Neurinomen sowie von anderen Tumoren ist. $\mathrm{Da}$ es aber wesentliche Unterschiede zwischen NF1 und NF2 gibt, ist eine separate Betrachtung notwendig.

NF1 ist eine häufige autosomal dominant vererbte Erkrankung mit einer Inzidenz von 1:3000, wesentlich seltener ist die ebenfalls dominant vererbte NF2 mit einer Inzidenz von 1:50.000. Die spontane Mutationsrate bei beiden Erkrankungen beträgt ca. 50\%, Männer und Frauen sind gleich häufig betroffen.

Charakteristisch für die NF1 ist die Bildung plexiformer Neurofibrome. Etwa $5-10 \%$ der plexiformen Neurofibrome kommen als pathognomonisch für NF1 vor, selten entarten sie maligne. Die Neurinome bei NF2 bestehen im Wesentlichen aus Schwann-Zellen ohne wesentliche Mukopolysaccharidmatrix. Diese Schwannome verdrängen die Nervenfaszikel eher als sie zu infiltrieren.

Bei der NF1 zeigt sich die Manifestation verstärkt an verschiedenen Organsystemen. Als erstes Zeichen der kutanen Manifestation sind die Pigmentstörungen der Haut, die als Café-au-lait-Flecken bezeichnet werden. Als weiteres Charakteristikum gilt das inguinale oder axilläre „freckling“. Ein typisches Merkmal sind auch die Knötchen der Iris, die als Hamartome gelten und bei 90\% der Erwachsenen mit Hilfe von Spaltlampenuntersuchungen nachweisbar sind. Hamartome der Retina sind seltener.
Ossäre Manifestationen sind z. B. die kongenitale laterale Tibiaverbiegung, eine knöcherne Dysplasie sowie das Vorkommen von Dextroskoliosen. Als vaskuläre Manifestation zeigen sich die fokalen oder bilateralen Dysplasien der Gefäße. Manifestationen am peripheren Nervensystem sind die kutanen Neurofibrome, die erst im Jugendalter auftreten und eher periphere Nerven betreffen. Die Spinalnerven sind dabei multipel betroffen. Bei etwa $3 \%$ aller NF1-Patienten entwickeln sich aus plexiformen Neurofibromen maligne Formen. Der häufigste Befund im MRT bei NF1-Patienten sind fokale Signalanhebungen in T2-gewichteten Sequenzen, die als so genannte Myelinvakuolisierung bezeichnet werden. Sie treten gehäuft in den Stammganglien, Thalamus, Hirnstamm und im Zerebellum auf. Insgesamt zeigt sich bei der Erkrankung ein erhöhtes Risiko für die Entstehung anderer Tumoren.

Die Neurofibromatose Typ 2 (NF2) ist klinisch charakterisiert durch einseitige Hörminderung im Erwachsenenalter als Erstsymptom, während Kinder im Falle einer Frühmanifestation mit multiplen Tumoren eher durch okuläre Katarakte auffällig werden. Insbesondere Kinder weisen häufig intrakranielle Meningeome oder spinale Tumoren auf. Kutane Schwannome kommen bei ca. $70 \%$ der Betroffenen vor, dabei handelt es sich in der Regel um intrakutane plaqueartige Läsionen. 70\% der Erwachsenen zeigen eine Polyneuropathie.

Das Hauptmerkmal der NF2 ist das bei über 90\% der Erwachsenen aufzufindende bilaterale vestibulare Schwannom. Dabei handelt es sich um gutartige Tumoren des superioren Astes des N. vestibularis. Tritt bei einem Kind ein Me- 
ningeom auf, liegt in $20 \%$ der Fälle eine NF2 vor. Spinale Tumoren kommen bei ca. $60-80 \%$ der Patienten vor, histologisch sind es meist Schwannome oder Meningeome. Zur Diagnosesicherung der NF2 sind neben der Erhebung der klinischen Historie und der Familienanamnese eine Untersuchung der Haut des Auges sowie der Neuroaxe und eine molekulare Analyse möglich.

Der Morbus Osler, auch als hereditäre hämorrhagische Teleangiektasie (HHT) bezeichnet, gehört ebenfalls zum Formenkreis der neurokutanen Symptome (Phakomatosen) und betrifft etwa 1:2500 bis 1:40.000 Menschen. Er gehört zur Gruppe der vaskulären hämorrhagischen Erkrankungen ohne zusätzliche Gerinnungsstörung. Dabei treten beim Morbus Osler rezidivierende spontane Epistaxien auf, daneben mukokutane Teleangiektasien. Die Ursache ist eine Veränderung in einem von mehreren HHT-assoziierten Genen. HHT-Patienten zeigen v. a. Teleangiektasien in der Nase und im Magen-DarmTrakt, auch arteriovenöse Malformationen häufig in Lunge, Gehirn und/oder Leber. Abhängig vom betroffenen Organsystem können unterschiedliche Symptome auftreten. Neben einer kardialen Belastung durch die arteriovenösen Shunts kann es zu bedrohlichen Blutungen und paradoxen Embolien, Schlaganfällen oder Hirnabszessen oder zur portalen Hypertension mit Umgehungskreisläufen kommen.

Die tuberöse Sklerose ist eine autosomal dominante Erkrankung mit Beteiligung des ZNS und anderer Organsysteme. Die Bildgebung ist für die Früherkennung von Komplikationen wichtig. Bei der tuberösen Sklerose handelt es sich um eine genetisch bedingte Multisystemerkrankung, die im Wesentlichen charakteristisch ist durch das Auftreten von Hamartomen in Gehirn, Herz, Haut, Auge, Niere, Lunge und Leber. Im Gehirn treten v. a. neben kortikalen und subkortikalen Tubera subependymale Riesenzellastrozytome (SEGA) auf. Von SEGA wird gesprochen, wenn eine subependymale Raumforderung einen Durchmesser von 1,3 cm überschreitet.

Das Sturge-Weber-Syndrom, auch als enzephalofaziale Angiomatose bezeichnet, ist gekennzeichnet durch einen Gesichts- nävus, Angiome der Hirnoberfläche, Hemiparese und fokale Krampfanfälle. Der Nävus ist tief rot und befindet sich im Versorgungsgebiet eines oder mehrerer Trigeminusäste. Die intrazerebralen gyralen Verkalkungen und angiomatösen Veränderungen können zu einem schweren fokalen Anfallsleiden führen. Diagnostisch stellen die intrakraniellen Verkalkungen die häufigste radiologische Manifestation des Sturge-Weber-Syndroms dar. Wichtig für den Verlauf der Erkrankung und die Therapie ist die Anfallsprophylaxe. Nicht selten werden bei diesen Patienten eine Hemisphärektomie bzw. eine Hemidekortikation, um eine Anfallsfreiheit oder Verminderung der Anfallshäufigkeit zu erreichen.

Wichtig erscheint, dass bei entsprechenden Veränderungen, v. a. an Haut und Augen, auch an das Vorliegen einer Phakomatose gedacht wird und entsprechende weiterführende Untersuchungen, v. a. des ZNS und anderer Organsysteme, durchgeführt werden.

Ihr

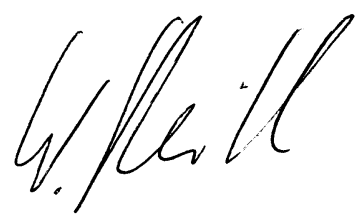

Prof. Dr. Wolfgang Reith

\section{Korrespondenzadresse}

\section{Prof. Dr. W. Reith}

Klinik für Diagnostische

und Interventionelle Neuroradiologie,

Universitätsklinikum des Saarlandes,

Kirrberger Straße, 66424 Homburg/Saar

wolfgang.reith@uniklinik-saarland.de

\section{Einhaltung ethischer Richtlinien}

Interessenkonflikt. W. Reith gibt an, dass kein Interessenkonflikt besteht.

\section{Deutsche Röntgengesellschaft informiert mit Wolfgang Nie- decken über den Schlaganfall} Zweite Ausgabe der Patientenzeitschrift "Medizin mit Durchblick" erschienen

Wie bringt man Patientinnen und Patienten die ganze Welt der Radiologie nahe - von den diagnostischen und therapeutischen Möglichkeiten bis hin zu den technischen Zusammenhängen? Und wie schafft man es dabei auch noch, ihnen im Wartezimmer eine fesselnde Lektüre zu bieten? Mit der neuen Ausgabe des Magazins „Medizin mit Durchblick" hat sich die Deutsche Röntgengesellschaft dieser anspruchsvollen Aufgabe gestellt. Als eine Weiterentwicklung der 2011 gestarteten Informationsinitiative will dieses Patientenmagazin beides: informieren und unterhalten. Entwickelt wurde das Magazin von professionellen Journalisten, die bewusst den Weg der publikumsnahen und personalisierten Darstellung der bildgebenden Medizin gegangen sind. Titelgeschichte der neuen Ausgabe ist die Geschichte von BAP-Sänger Wolfang Niedecken, der dank rascher Behandlung in der Neuroradiologie des Kölner Universitätsklinikums den Schlaganfall überlebt hat.

Der Charme des Magazins liegt darin, dass nicht die medizinischen Zusammenhänge, sondern die Geschichten der Patienten im Vordergrund stehen; ,"Medizin mit Durchblick" erklärt nicht nur, es erzählt Geschichten aus der Radiologie.

Neben der Titelgeschichte um den „kölschen Bob Dylan" geht es in Heft 2 um Früherkennung von Krankheiten, zum Beispiel Osteoporose, um den Beruf der Medizinisch-technischen Radiologie-Assistenten und die Geschichte der Kinderradiologie, die dieses Jahr ihren 50. Geburtstag in Deutschland begeht. Das Patientenmagazin kann über www.drg.de bestellt werden, ferner ist auf dieser Seite eine e-paper-Fassung des Magazins abrufbar.

Quelle:

Deutsche Röntgengesellschaft e.V. www.drg.de www.medizin-mit-durchblick.de 